\title{
Experimental demonstration of a universally valid error-disturbance uncertainty relation in spin measurements
}

\author{
Jacqueline Erhart', Stephan Sponar', Georg Sulyok', Gerald Badurek, Masanao Ozawa² \\ and Yuji Hasegawa ${ }^{1 \star}$
}

The uncertainty principle generally prohibits simultaneous measurements of certain pairs of observables and forms the basis of indeterminacy in quantum mechanics'. Heisenberg's original formulation, illustrated by the famous $\gamma$-ray microscope, sets a lower bound for the product of the measurement error and the disturbance ${ }^{2}$. Later, the uncertainty relation was reformulated in terms of standard deviations $s^{3-5}$, where the focus was exclusively on the indeterminacy of predictions, whereas the unavoidable recoil in measuring devices has been ignored ${ }^{6}$. A correct formulation of the error-disturbance uncertainty relation, taking recoil into account, is essential for a deeper understanding of the uncertainty principle, as Heisenberg's original relation is valid only under specific circumstances $^{7-10}$. A new error-disturbance relation, derived using the theory of general quantum measurements, has been claimed to be universally valid ${ }^{11-14}$. Here, we report a neutronoptical experiment that records the error of a spin-component measurement as well as the disturbance caused on another spin-component. The results confirm that both error and disturbance obey the new relation but violate the old one in a wide range of an experimental parameter.

The uncertainty relation was first proposed by Heisenberg ${ }^{2}$ in 1927 as a limitation of simultaneous measurements of canonically conjugate variables owing to the back-action of the measurement: the measurement of the position $Q$ of the electron with the error $\epsilon(Q)$, or 'the mean error', induces the disturbance $\eta(P)$, or 'the discontinuous change', of the momentum $P$ so that they always satisfy the relation

$$
\epsilon(Q) \eta(P) \sim \frac{\hbar}{2}
$$

where $\hbar$ is Planck's constant divided by $2 \pi$ (here, we use $\hbar / 2$ for consistency with modern treatments). In a mathematical derivation of the above relation from the commutation relation $Q P-P Q=i \hbar$, Heisenberg $^{2}$ used the reciprocal relation $\sigma(Q) \sigma(P) \geq \hbar / 2$ for standard deviations $\sigma(Q), \sigma(P)$ of position and momentum, which was proved shortly afterwards in ref. 3 for arbitrary wavefunctions. This relation was generalized to arbitrary pairs of observables $A$, $B$ by Robertson ${ }^{4}$ as

$$
\sigma(A) \sigma(B) \geq \frac{1}{2}|\langle\psi|[A, B]| \psi\rangle|
$$

in any states $|\psi\rangle$ with $\sigma(A), \sigma(B)<\infty$. Here, $[A, B]$ represents the commutator $[A, B]=A B-B A$ and the standard deviation is defined as $\sigma(A)^{2}=\left\langle\psi\left|A^{2}\right| \psi\right\rangle-\langle\psi|A| \psi\rangle^{2}$. Note that a positive definite covariance term can be added to the right-hand side of equation (2), if squared, as discussed by Schrödinger ${ }^{5}$. For our experimental setting, this term vanishes. Robertson's relation (equation (2)) for standard deviations has been confirmed by many different experiments. In a single-slit diffraction experiment ${ }^{15}$ the uncertainty relation, as expressed in equation (2), has been confirmed. A trade-off relation appears in squeezing coherent states of radiation fields ${ }^{16}$, and many experimental demonstrations have been carried out ${ }^{17}$.

Robertson's relation (equation (2)) has a mathematical basis, but has no immediate implications for limitations on measurements. This relation is naturally understood as limitations on state preparation or limitations on prediction from the past. On the other hand, the proof of the reciprocal relation for the error $\epsilon(A)$ of an $A$ measurement and the disturbance $\eta(B)$ on observable $B$ caused by the measurement, in a general form of Heisenberg's error-disturbance relation

$$
\epsilon(A) \eta(B) \geq \frac{1}{2}|\langle\psi|[A, B]| \psi\rangle|
$$

is not straightforward, as Heisenberg's proof ${ }^{2}$ used an unsupported assumption on the state just after the measurement ${ }^{12}$, despite successful justifications for the Heisenberg-type relation for unbiased joint measurements ${ }^{8-10}$. Recently, rigorous and general theoretical treatments of quantum measurements have revealed the failure of Heisenberg's relation (equation (1)), and derived a new universally valid relation ${ }^{11-14}$ given by

$$
\epsilon(A) \eta(B)+\epsilon(A) \sigma(B)+\sigma(A) \eta(B) \geq \frac{1}{2}|\langle\psi|[A, B]| \psi\rangle|
$$

Here, the error $\epsilon(A)$ is defined as the root mean squared (r.m.s.) of the difference between the output operator $O_{A}$ actually measured and the observable $A$ to be measured, whereas the disturbance $\eta(B)$ is defined as the r.m.s. of the change in observable $B$ during the measurement ${ }^{11,13}$ (see Methods, 'Universally valid uncertainty relation', for details). The additional second and third terms result mathematically from non-commutativity between $B(A)$ and the error (disturbance) operator (Equation (235) in ref. 13) and imply a new accuracy limit $\epsilon(A) \geq(1 / 2)|\langle\psi|[A, B]| \psi\rangle| \sigma(B)^{-1}$ for non-disturbing $(\eta(B)=0)$ measurements and a new disturbance limit $\eta(B) \geq(1 / 2)|\langle\psi|[A, B]| \psi\rangle| \sigma(A)^{-1}$ for noise-free $(\epsilon(A)=0)$ measurements, instead of $\epsilon(A) \sim \infty$ or $\eta(B) \sim \infty$ as derived from the Heisenberg-type relation (equation (3)). Note that the above relations (equations (3) and (4)) are based on the state- 


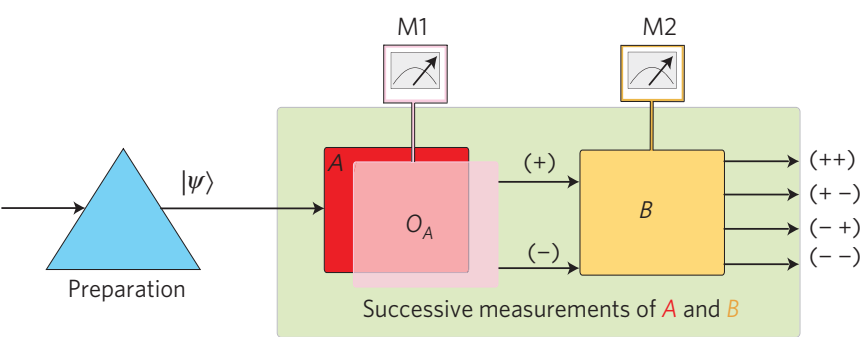

Figure 1 Concept of the experiment. A successive measurement scheme of observables $A$ and $B$ is exploited for the demonstration of the error-disturbance uncertainty relation. After preparing an initial state $|\psi\rangle$, apparatus $\mathrm{M} 1$ is assumed to measure an observable $A$ (red region). The error $\epsilon(A)$ and the disturbance $\eta(B)$ are experimentally controlled by detuning apparatus $\mathrm{M} 1$ to make a projective measurement of $O_{A}$ instead of $A$ (light red). The disturbance $\eta(B)$ on the observable $B$ is caused by the measurement made by apparatus $M 1$, which randomly projects the state onto one of the eigenstates of $O_{A}$, and is quantified using the $B$ measurement carried out by apparatus $M 2$ (yellow region). The successive measurements of $O_{A}$ and $B$ result in four possible outcomes, denoted as $(++),(+-),(-+)$ and $(--)$, from which error $\epsilon(A)$ and disturbance $\eta(B)$ are quantitatively determined.

dependent formulation of error and disturbance for arbitrary observables. For the position-momentum case, there are different approaches based on the state-independent formulation ${ }^{18-20}$, where the lower bound for error-disturbance inequality depends strongly on the measures used.

We have experimentally tested the universally valid errordisturbance relation (equation (4)) for neutron-spin measurements ${ }^{2}$
We set $A$ and $B$ as the $x$ and $y$ components of the neutron $1 / 2$ spin. (For simplicity, $\hbar / 2$ is omitted for each spin component.) The error $\epsilon(A)$ and the disturbance $\eta(B)$ are defined for a measuring apparatus called $\mathrm{M} 1$, so that apparatus $\mathrm{M} 1$ measures the observable $A=\sigma_{x}$ with error $\epsilon(A)$ and disturbs the observable $B=\sigma_{y}$ with disturbance $\eta(B)$ during the measurement, where $\sigma_{x}$ and $\sigma_{y}$ denote the Pauli matrices. To control the error $\epsilon(A)$ and the disturbance $\eta(B)$, apparatus $\mathrm{M} 1$ is designed to actually carry out the projective measurement of $O_{A}=\sigma_{\phi}=\sigma_{x} \cos \phi+\sigma_{y} \sin \phi$ instead of exactly measuring $A=\sigma_{x}$ by detuning the azimuthal angle $\phi$ of $\sigma_{\phi}$ between 0 and $\pi / 2$, which is an experimentally controlled parameter, so that $\epsilon(A)$ and $\eta(B)$ are determined as a function of $\phi$ (see Methods for details). As the output operator $O_{A}$ and the observable $A$ to be measured are not simultaneously measurable, their difference is not a directly detectable quantity, and neither is the change of the observable $B$. On this ground, the notions of the error $\epsilon(A)$ and the disturbance $\eta(B)$ have been often claimed to be experimentally inaccessible ${ }^{19,22}$. To overcome this difficulty, we use the method proposed in ref. 13 to determine the error $\epsilon(A)$ and the disturbance $\eta(B)$ from experimentally available data (see Methods for details). A different method for the experimental demonstration of the same relation (equation (4)), which exploits the weak-measurement technique used for measuring momentum transfer in ref. 23, was recently proposed in ref. 24 .

In our experiment, a neutron beam passes a preparation stage of the initial state $|\psi\rangle$. The error $\epsilon(A)$ is determined by the data from the apparatus $\mathrm{M} 1$, and the disturbance $\eta(B)$ is determined by the data from another apparatus called M2, which carries out the projective measurement of $B$ on the state just after the M1 measurement. Thus, the experiment is based on the successive projective measurements of two non-commuting

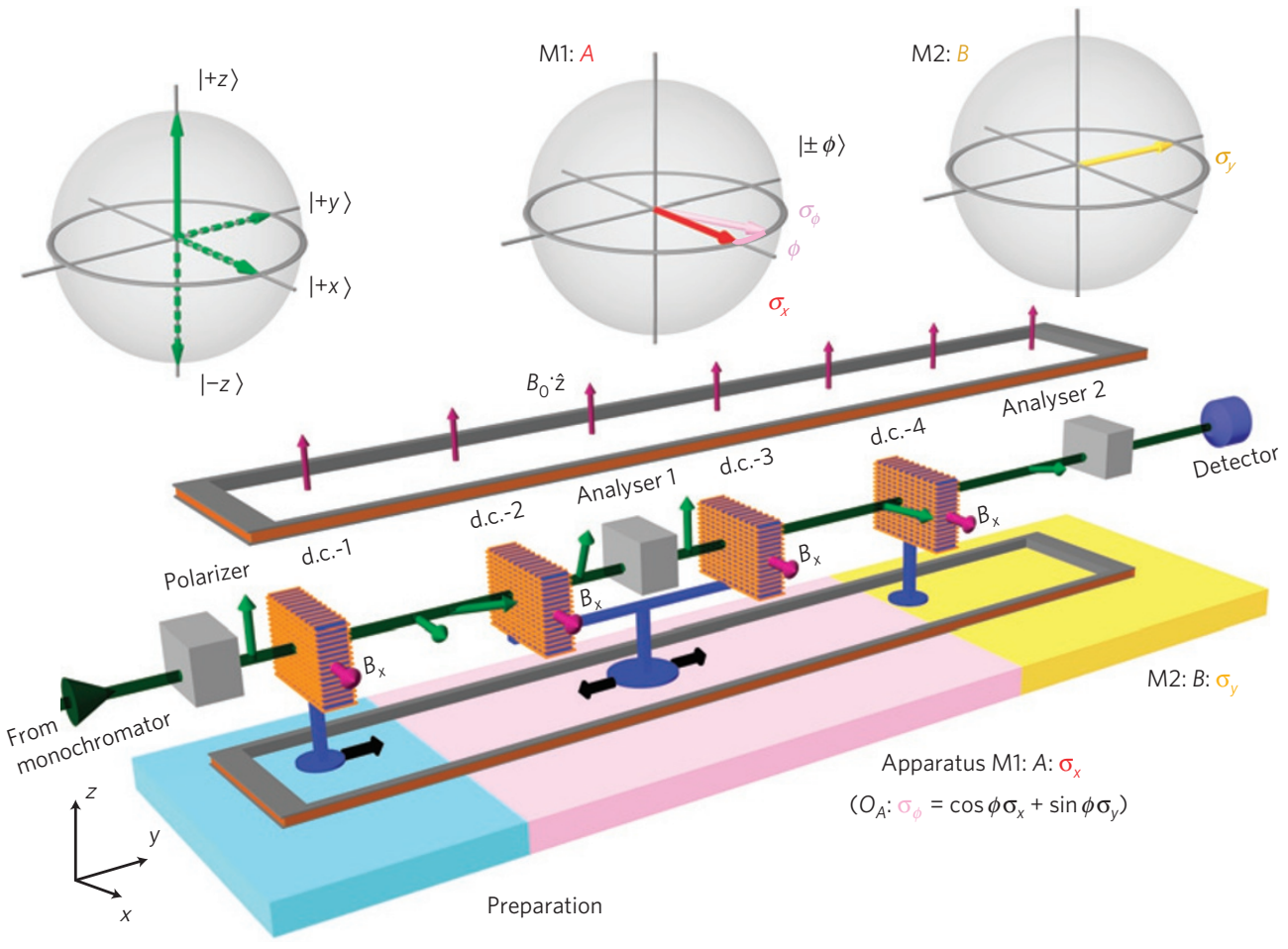

Figure 2 | Illustration of the experimental apparatus. The set-up is designed for demonstration of the universally valid uncertainty relation for error and disturbance in neutron spin measurements. The neutron optical set-up consists of three stages: preparation (blue region), apparatus M1 making the measurement of observable $\mathrm{O}_{A}=\sigma_{\phi}$ (red region) and apparatus $\mathrm{M} 2$ carrying out the measurement of observable $B=\sigma_{y}$ (yellow region). A monochromatic neutron beam is polarized in the $+z$ direction by passing through a supermirror spin polarizer. By combining the action of four $d$.c. coils, the magnetic guide field $B_{0}$ and the analysing supermirrors, the successive measurements of $\sigma_{\phi}$ and $\sigma_{y}$ are made for the required states (see Methods for details). The error $\epsilon(A)$ and the disturbance $\eta(B)$, as well as the standard deviations of $\sigma(A)$ and $\sigma(B)$, are determined from the expectation values of the successive measurements. 


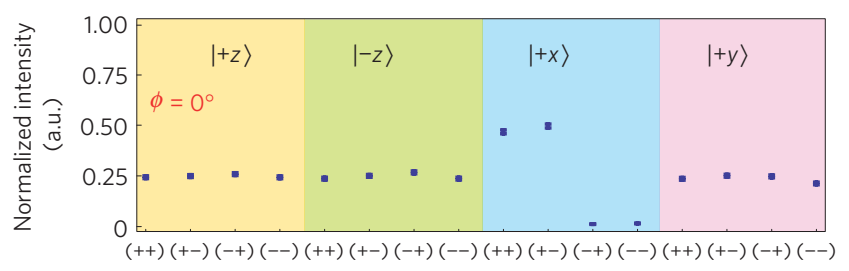

Combined outcomes of $\mathrm{M} 1$ and $\mathrm{M} 2$

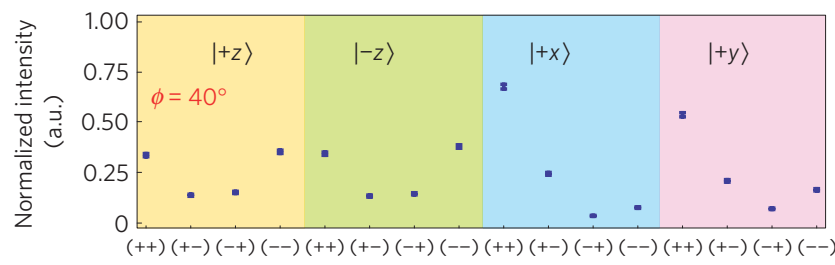

Combined outcomes of $M 1$ and $M 2$

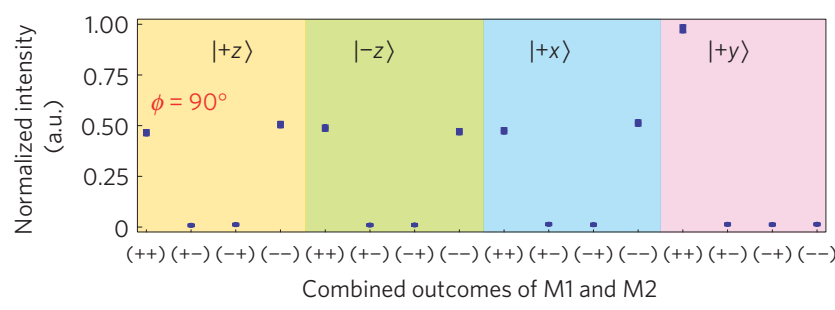

Figure 3 | Experimental results. Normalized intensity of the successive measurements carried out by apparatus $\mathrm{M} 1$ and $\mathrm{M} 2$. The successive measurements of $\mathrm{M} 1$ and $\mathrm{M} 2$ have four outcomes, denoted as $(++)$, $(+-),(-+)$ and $(--)$. Intensities, according to the corresponding outcomes, are depicted for each initial state, that is $|+z\rangle,|-z\rangle,|+x\rangle$ and $|+y\rangle$. Three sets for detuning parameter $\phi=0^{\circ}, 40^{\circ}$ and $90^{\circ}$ are plotted. The error $\epsilon(A)$ and the disturbance $\eta(B)$ are determined from these 16 intensities, for each setting of the detuning parameter $\phi$. Error bars represent \pm 1 s.d. of the normalized intensities. Some error bars are smaller than the size of the markers.

observables $O_{A}$ in $\mathrm{M} 1$ and $B$ in $\mathrm{M} 2$ as depicted in Fig. 1. The apparatus $\mathrm{M} 1$ has two possible outcomes, that is +1 and -1 , corresponding to measurement operators $E^{\phi}( \pm 1)=(1 / 2)\left(I \pm \sigma_{\phi}\right)$. The apparatus M2 also yields either +1 or -1 , corresponding to measurement operators $E^{y}( \pm 1)=(1 / 2)\left(I \pm \sigma_{y}\right)$. Thus, the successive measurements carried out by M1 and M2 finally result in four intensities denoted as $(++),(+-),(-+)$ and $(--)$. The set-up of the neutron spin experiment is depicted in Fig. 2. In the actual experiment the spin analysers select only one spin component. Hence, the four possible outcomes are recorded not at the same time, as in an ideal projective measurement, but one after the other. As seen from equations (7) and (8), error $\epsilon(A)$ and disturbance $\eta(B)$ for the state $|\psi\rangle=|+z\rangle$ are obtained by making the successive measurements of $O_{A}$ and $B$ on the state $|\psi\rangle=|+z\rangle$ as well as the auxiliary states $|-z\rangle$, $|+x\rangle$ and $|+y\rangle$.

The experiment was carried out at the research reactor facility TRIGA Mark II of the Vienna University of Technology. The monochromatic neutron beam with a mean wavelength of $1.96 \AA$ propagates in the $+y$ direction. The beam is approximately $99 \%$ polarized crossing a bent $\mathrm{Co}-\mathrm{Ti}$ supermirror array (polarizer) ${ }^{25}$. Two analysing supermirrors (analysers) are adjusted to higher incident angles so that the second-order harmonics in the incident beam are suppressed. The final intensity was about 90 neutrons $\mathrm{s}^{-1}$ at a beam cross-section of 10 (vertical) $\times 5$ (horizontal) $\mathrm{mm}^{2}$. A ${ }^{3} \mathrm{He}$ monitor detector is used for normalization to correct statistical

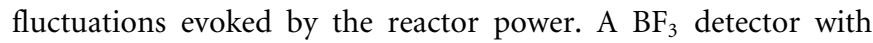
high efficiency (more than 99\%) is used for the experiment. To avoid unwanted depolarization, a static guide field pointing in
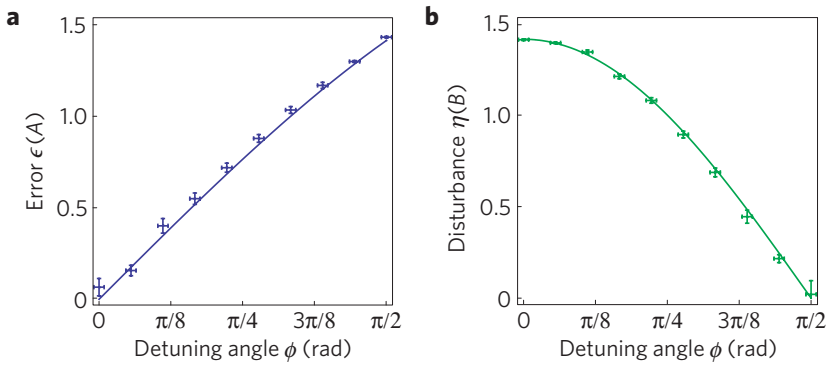

Figure 4 | Trade-off relation between error and disturbance. a,b, Error $\epsilon(A)$ (a) and disturbance $\eta(B)(\mathbf{b})$ both as a function of the detuning angle $\phi$ with the plots of the values determined by the experiment. The theory predicts $\epsilon(A)=2 \sin (\phi / 2)$ and $\eta(B)=\sqrt{2} \cos \phi$. The experimental data are in good agreement with theory, showing the trade-off: the less the error, the more the disturbance. Error bars represent \pm 1 s.d.

the $+z$ direction with a strength of about 10 Gauss permeates rectangular Helmholtz coils. In addition, the guide field induces Larmor precession, which, together with four appropriately placed d.c. spin rotator coils, enables state preparation and projective measurements of $O_{A}$ in M1 and $B$ in M2 (see Methods for details).

To test the universally valid uncertainty relation stated in equation (4), the standard deviations $\sigma(A)$ and $\sigma(B)$, the error $\epsilon(A)$ and the disturbance $\eta(B)$ are determined. The measurements of the standard deviations $\sigma(A)$ and $\sigma(B)$ are carried out by M1 and M2 separately, whereas error $\epsilon(A)$ and disturbance $\eta(B)$ are determined by successive projective measurements using M1 and M2.

Typical experimental data sets, for miscellaneous detuning angles $\phi$, are depicted in Fig. 3. The resulting values of $\epsilon(A)$ and $\eta(B)$, together with the theoretical predictions $\epsilon(A)=2 \sin (\phi / 2)$ and $\eta(B)=\sqrt{2} \cos \phi$ (Methods), are plotted as a function of the detuning parameter $\phi$ in Fig. 4. The trade-off relation of $\epsilon(A)$ and $\eta(B)$ is in good agreement with theory: when one observable is measured more precisely, the other is more disturbed. The calculated expectation values are normalized owing to the limiting efficiency of the entire apparatus $(\sim 96 \%)$, so that expectation values are bounded between \pm 1 (Methods). Errors are calculated using error propagation from the standard deviation of the count rates and considering inaccuracies of the Larmor precession angles $\left(\sim 1.5^{\circ}\right)$ : the latter stem mainly from inhomogeneity of the guide field along the beam.

From the terms obtained above (error $\epsilon(A)$, disturbance $\eta(B)$, standard deviations $\sigma(A)$ and $\sigma(B))$, the Heisenberg errordisturbance product $\epsilon(A) \eta(B)$ and the universally valid expression $\epsilon(A) \eta(B)+\epsilon(A) \sigma(B)+\sigma(A) \eta(B)$, that is, the left-hand side of (equation (4)), are plotted as a function of the detuned azimuthal angle $\phi$ in Fig. 5a. This figure illustrates the fact that the Heisenberg product is always below the calculated limit, and that the universally valid expression is always larger than the limit in the scanned range of $\phi$.

The invalidity of the naive Heisenberg limit for projective measurements of bounded observables, as in the present experiment, has been discussed in ref. 14. In fact, the projective measurements of $A$ and $B$ achieve $\epsilon(A)=0$ and $\eta(B)=0$, respectively, whereas we have $0 \leq \eta(B), \epsilon(A) \leq 2$ for any measurement. Thus, at least some region around these points $(\phi=0$ and $\phi=\pi / 2)$ is expected to exhibit the violation of equation (3). Moreover, owing to our choice of observables and the initial state, we keep the Heisenberg product $\epsilon(A) \eta(B)$ below the limit throughout the entire range of $\phi$.

Although the error is experimentally controlled by a single parameter here, it is easily extendable for uncontrolled and fluctuating parameters. Here, we concentrate on the situation where the full trade-off relation between error $\epsilon(A)$ and disturbance $\eta(B)$ occurs. It is worth noting that the mean value of the observable 


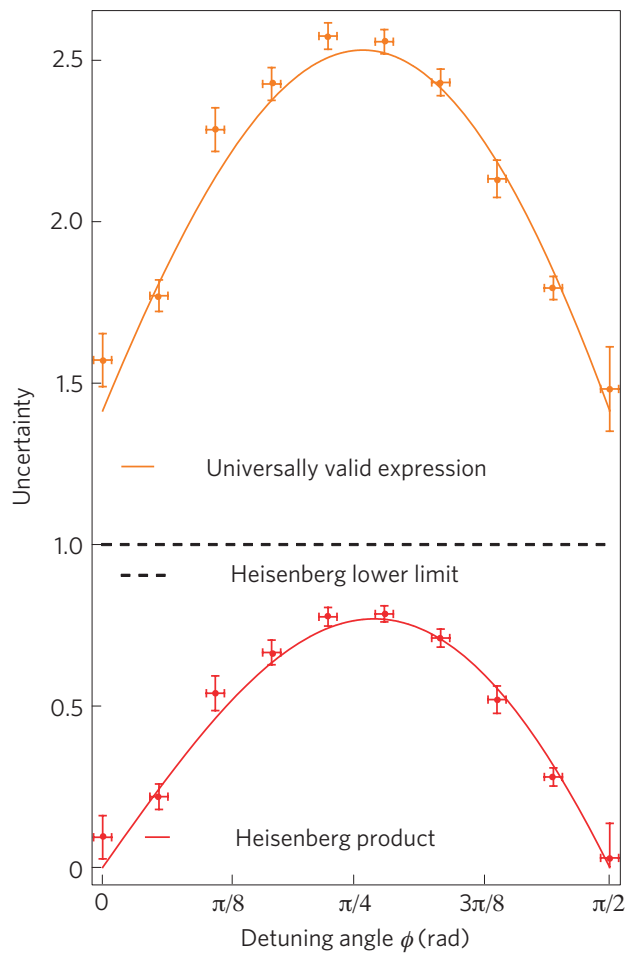

b

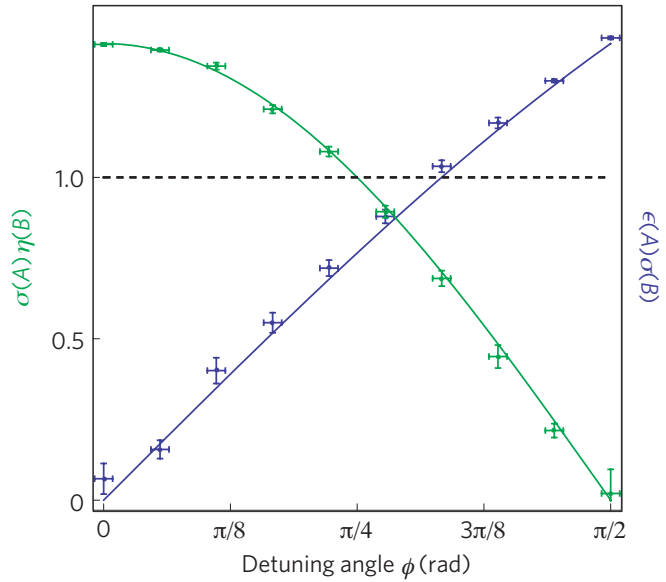

Figure 5 | Experimentally determined values of the universally valid uncertainty relation. $\mathbf{a}$, The universally valid expression

$\epsilon(A) \eta(B)+\epsilon(A) \sigma(B)+\sigma(A) \eta(B)$ (orange) and the actual Heisenberg product $\epsilon(A) \eta(B)$ (red) as a function of the detuning angle $\phi$ with theoretical predictions. The Heisenberg product is always smaller than the calculated limit $(1 / 2)|\langle\psi|[A, B]| \psi\rangle|=1$ (depicted as the dashed line). In contrast, the universally valid expression is always larger than the limit. b, The two additional product terms $\sigma(A) \eta(B)$ (green) and $\epsilon(A) \sigma(B)$ (blue) in the universally valid expression together with the theoretical predictions. $\epsilon(A), \eta(B)$ and $\sigma(A)(\sigma(B))$ represent error, disturbance and standard deviations, respectively. Error bars represent \pm 1 s.d.

$A$ is correctly reproduced for any detuning angle $\phi$, that is, $\left\langle+z\left|O_{A}\right|+z\right\rangle=\langle+z|A|+z\rangle$, so that the projective measurement of $O_{A}$ reproduces the correct probability distribution of $A$, whereas we can detect the non-zero r.m.s. error $\epsilon(A)$ for $\phi \neq 0$. What has been shown by the uncertainty principle is the existence of an unavoidable trade-off between measurement accuracy and disturbance, but this principle has eluded a satisfactory quantitative description for a long time. Our demonstration is the first evidence for the validity of the new relation (equation (4)) proposed as a universally valid error-disturbance relation ${ }^{11-14}$; moreover the failure of the old relation (equation (3)) is also illustrated. Our result demonstrates that the new relation solves a long-standing problem of describing the relation between measurement accuracy and disturbance, and sheds light on fundamental limitations of quantum measurements, for instance on the debate of the standard quantum limit for monitoring free-mass position ${ }^{26-28}$.

\section{Methods}

Universally valid uncertainty relation. Any measuring apparatus $\mathbf{M}$ is, in principle, modelled by the unitary operator $U$ describing the time evolution of the composite system of the measured object $\mathbf{S}$ and the probe system $\mathbf{P}$ during the measuring interaction and the meter observable $M$ of $\mathbf{P}$ actually measured after the measuring interaction ${ }^{13}$. If the initial states of the object and the apparatus are $|\psi\rangle$ and $|\xi\rangle$, respectively, the r.m.s. error $\epsilon(A)$ of $\mathbf{M}$ for measuring an observable $A$ of $\mathbf{S}$ and the r.m.s. disturbance $\eta(B)$ of $\mathbf{M}$ caused on an observable $B$ of $\mathbf{S}$ are defined as

$$
\begin{gathered}
\epsilon(A)=\|\left[U^{\dagger}(I \otimes M) U-A \otimes I\right]|\psi\rangle|\xi\rangle \| \\
\eta(B)=\|\left[U^{\dagger}(B \otimes I) U-B \otimes I\right]|\psi\rangle|\xi\rangle \|
\end{gathered}
$$

where $\|\cdots\|$ denotes the norm of the state vector and $I$ stands for the identity operator. Then, it is mathematically proved ${ }^{11,13}$ that equation (4) holds for any unitary operator $U$ for $\mathbf{S}+\mathbf{P}$, observable $M$ of $\mathbf{P}$ and state vectors $|\psi\rangle$ of $\mathbf{S}$ and $|\xi\rangle$ of $\mathbf{P}$. Suppose that the apparatus $\mathbf{M}$ has a family $\left\{M_{\mathrm{m}}\right\}$ of measurement operators ${ }^{29}$. This means that the measuring apparatus $\mathbf{M}$ has possible outcomes $m$ with probability $p(m)=\| M_{\mathrm{m}}|\psi\rangle \|^{2}$ and the state of the object $\mathbf{S}$ after the measurement with outcome $m$ is $M_{\mathrm{m}}|\psi\rangle / \| M_{\mathrm{m}}|\psi\rangle \|$. In this case, the r.m.s. error and r.m.s. disturbance are given by ${ }^{14}$

$$
\begin{gathered}
\epsilon(A)^{2}=\sum_{\mathrm{m}} \| M_{\mathrm{m}}(m-A)|\psi\rangle \|^{2} \\
\eta(B)^{2}=\sum_{\mathrm{m}} \|\left[M_{\mathrm{m}}, B\right]|\psi\rangle \|^{2}
\end{gathered}
$$

If $\left\{M_{\mathrm{m}}\right\}$ consists of mutually orthogonal projections, the measurement is called a projective measurement. In this case, equation (5) can be simplified as $\epsilon(A)=\|\left(O_{A}-A\right)|\psi\rangle \|$ by the Pythagorean theorem, where $O_{A}=\sum_{\mathrm{m}} m M_{\mathrm{m}}$ is called the output operator.

Error and disturbance in spin measurements: theoretical determination. In the experiment, we test the universally valid uncertainty relation (equation (4)) for observables $A=\sigma_{x}$ and $B=\sigma_{y}$, where the initial state $|\psi\rangle$ is $|+z\rangle$ and the measuring apparatus $\mathbf{M}=\mathrm{M} 1$ is considered to carry out the projective measurement of $O_{A}=\sigma_{\phi}=\cos \phi \sigma_{x}+\sin \phi \sigma_{y}$. Thus, the apparatus M1 is described by measurement operators $E^{\phi}(+1)=\left(1+\sigma_{\phi}\right) / 2$ and $E^{\phi}(-1)=\left(1-\sigma_{\phi}\right) / 2$ with $O_{A}=\sum_{x= \pm 1} x E^{\phi}(x)$. From equations (5) and (6), we have

$$
\begin{gathered}
\epsilon(A)=\|\left(\sigma_{\phi}-\sigma_{x}\right)|\psi\rangle \|=2 \sin \frac{\phi}{2} \\
\eta(B)=\sqrt{2} \|\left[\sigma_{\phi} / 2, \sigma_{y}\right]|\psi\rangle \|=\sqrt{2} \cos \phi
\end{gathered}
$$

Experimental determination of error and disturbance. In the experiment, we determine $\epsilon(A)$ and $\eta(B)$ from statistically available data obtained by successive neutron spin measurements. According to the former theoretical analysis (refs. 13, p. 387), the error $\epsilon(A)$ is determined by mean values of $O_{A}$ in three different states as

$$
\begin{aligned}
\epsilon(A)^{2}= & \left\langle\psi\left|A^{2}\right| \psi\right\rangle+\left\langle\psi\left|O_{A}^{2}\right| \psi\right\rangle+\left\langle\psi\left|O_{A}\right| \psi\right\rangle+\left\langle\psi\left|A O_{A} A\right| \psi\right\rangle \\
& -\left\langle\psi\left|(A+I) O_{A}(A+I)\right| \psi\right\rangle \\
= & 2+\left\langle\psi\left|O_{A}\right| \psi\right\rangle+\left\langle A \psi\left|O_{A}\right| A \psi\right\rangle-\left\langle(A+I) \psi\left|O_{A}\right|(A+I) \psi\right\rangle
\end{aligned}
$$

where we have used the following abbreviations: $|A \psi\rangle=A|\psi\rangle$ and $|(A+I) \psi\rangle=(A+I)|\psi\rangle$. As apparatus M1 carries out the projective measurement of $O_{A}$, to determine $\epsilon(A)$ for the basic initial state $|\psi\rangle$ we need only to measure the intensities from apparatus M1 in the three auxiliary incident states of M1 corresponding to $|\psi\rangle, A|\psi\rangle,(A+I)|\psi\rangle$. The expectation values expressed in equation (7) are calculated from the measured intensities, depicted in Fig. 3, using

$$
\left\langle\psi\left|O_{A}\right| \psi\right\rangle=\frac{\left(I_{++}+I_{+-}\right)-\left(I_{-+}+I_{--}\right)}{I_{++}+I_{+-}+I_{-+}+I_{--}}
$$

To detect the disturbance on $B$ caused by apparatus M1, apparatus M2 carries out the projective measurement of $B$ in the state just after the M1 measurement. The modified output operators of apparatus $\mathrm{M} 2$ for the initial state of $\mathrm{M} 1$ are given by $O_{B}=\sum_{x} E^{\phi}(x) B E^{\phi}(x)$ and $O_{B}^{(2)}=\sum_{x} E^{\phi}(x) B^{2} E^{\phi}(x)$, which describe the mean 
and the second moment of the M2 measurement for the initial state of M1. Then, from Eqs. (189) and (227) of ref. 13 the disturbance $\eta(B)$ is also determined by mean values of $O_{B}$ in three different states as

$$
\begin{aligned}
\eta(B)^{2}= & \left\langle\psi\left|B^{2}\right| \psi\right\rangle+\left\langle\psi\left|O_{B}^{(2)}\right| \psi\right\rangle+\left\langle\psi\left|O_{B}\right| \psi\right\rangle+\left\langle B \psi\left|O_{B}\right| B \psi\right\rangle \\
& -\left\langle(B+I) \psi\left|O_{B}\right|(B+I) \psi\right\rangle \\
= & 2+\left\langle\psi\left|O_{B}\right| \psi\right\rangle+\left\langle B \psi\left|O_{B}\right| B \psi\right\rangle-\left\langle(B+I) \psi\left|O_{B}\right|(B+I) \psi\right\rangle
\end{aligned}
$$

where the similar notations $|B \psi\rangle$ and $|(B+I) \psi\rangle$ are used as before and the expectation values are given by

$$
\left\langle\psi\left|O_{B}\right| \psi\right\rangle=\frac{\left(I_{++}+I_{-+}\right)-\left(I_{+-}+I_{--}\right)}{I_{++}+I_{+-}+I_{-+}+I_{--}}
$$

Thus $\eta(B)$ is determined in the same manner as $\epsilon(A)$. By the relations $|\psi\rangle=|+z\rangle$, $A|\psi\rangle=|-z\rangle, B|\psi\rangle=i|-z\rangle,(A+I)|\psi\rangle=\sqrt{2}|+x\rangle$, and $(B+I)|\psi\rangle=\sqrt{2}|+y\rangle$, where we set $|+x\rangle=(|+z\rangle+|-z\rangle) / \sqrt{2}$ and $|+y\rangle=(|+z\rangle+i|-z\rangle) / \sqrt{2}$, the required states in equations (7) and (8) are generated by spinor rotations in the experiment (Fig. 2); the normalization factors are confirmed experimentally in spin-rotation measurement. Note that for a general observable $X$ the state $X|\psi\rangle$ can be prepared with success probability $\| X|\psi\rangle\left\|^{2} /\right\| X \|^{2}$ by applying a 'completely positive operation ${ }^{30}$. Thus, the states $A|\psi\rangle,(A+I)|\psi\rangle, B|\psi\rangle$ and $(B+I)|\psi\rangle$ can be prepared in principle without knowing what the state $|\psi\rangle$ is.

State preparation and successive neutron spin measurements. By using a supermirror polarizer, the incoming monochromatic neutron beam $(\Delta \lambda / \lambda \simeq 0.02)$ is highly polarized ( $\sim 99 \%)$ in the $+z$ direction. The state of the neutrons is controlled basically by four d.c. coil spin-turners (Fig. 2). The first d.c. coil (d.c.-1) produces a magnetic field in the $x$ direction $\left(B_{x}\right)$, which can be used to rotate the inital polarization vector about the $x$ axis. By further exploiting Larmor precession about the $+z$ axis, induced by the static guide field present throughout the entire set-up, and varying the position of d.c.-1, arbitrary initial states can be produced at the end of the preparation stage (up to an irrelevant phase factor). In our particular configuration the required states are $|+z\rangle,|-z\rangle,|+y\rangle$ and $|+x\rangle$. For the generation of $|+z\rangle$ d.c. -1 is switched off, whereas the spin is flipped by d.c. -1 for $|-z\rangle$ preparation. The state $|+y\rangle$ is generated by applying a $\pi / 2$ rotation around the $x$ axis and the $|+x\rangle$ state is produced by further moving the position of d.c.- 1 by one-quarter of the Larmor rotation period. The projective measurement of observable $O_{A}$ is realized by similar mechanisms: the prepared state rotates about the $z$ axis owing to Larmor precession. Hence, by properly placing the d.c.-2 coil, the spin component to be measured can be projected towards the $+z$ direction, where it is reflected by the supermirror analyser-1. After passing through this first analyser in the $+\mathrm{z}$ state, d.c.-3 produces the eigenstate $| \pm \phi\rangle=E^{\phi}( \pm 1)|\psi\rangle$ of $\sigma_{\phi}$ so that the apparatus M1 makes the projective measurement of $O_{A}$ to obtain the mean values of $O_{A}$ in equation (7). In the same manner, apparatus M2 makes the measurement of observable $B$ on the eigenstate $| \pm \phi\rangle$, which detects the disturbance on the observable $B$. Hence we obtain the mean values of $O_{B}$ in equation (8) from apparatus M2. The combination of the projective measurements of $\sigma_{\phi}$ and $\sigma_{y}$ gives four count rates at the neutron detector in the downstream of the beam. Note that the measurement made by apparatus M2 is the error-free $B$ measurement on the state just after the measurement carried out by M1.

Received 18 May 2011; accepted 5 December 2011; published online 15 January 2012; corrected online 12 March 2012 and 1 August 2012

\section{References}

1. Wheeler, J. A. \& Zurek, W. H. (eds) in Quantum Theory and Measurement (Princeton Univ. Press, 1983).

2. Heisenberg, W. Über den anschaulichen Inhalt der quantentheoretischen Kinematik und Mechanik. Z. Phys. 43, 172-198 (1927).

3. Kennard, E. H. Zur Quantenmechanik einfacher Bewegungstypen. Z. Phys. 44, 326-352 (1927).

4. Robertson, H. P. The uncertainty principle. Phys. Rev. 34, 163-164 (1929).

5. Schrödinger, E. Zum Heisenbergschen Unschärfeprinzip. Sitz. Preuss. Akad. Wiss. 14, 296-303 (1930).
6. Ballentine, L. E. The statistical interpretation of quantum mechanics. Rev. Mod. Phys. 42, 358-381 (1970).

7. Arthurs, E. \& Kelly, J. L. Jr On the simultaneous measurement of a pair of conjugate observables. Bell Syst. Tech. J. 44, 725-729 (1965).

8. Arthurs, E. \& Goodman, M. S. Quantum correlations: A generalized Heisenberg uncertainty relation. Phys. Rev. Lett. 60, 2447-2449 (1988).

9. Ishikawa, S. Uncertainty relations in simultaneous measurements for arbitrary observables. Rep. Math. Phys. 29, 257-273 (1991).

10. Ozawa, M. in Quantum Aspects of Optical Communications. (eds Bendjaballah, C., Hirota, O. \& Reynaud, S.) (Lecture Notes in Physics, Vol. 378, 3-17, Springer, 1991).

11. Ozawa, M. Universally valid reformulation of the Heisenberg uncertainty principle on noise and disturbance in measurements. Phys. Rev. A 67, 042105 (2003).

12. Ozawa, M. Physical content of the Heisenberg uncertainty relation: Limitation and reformulation. Phys. Lett. A 318, 21-29 (2003).

13. Ozawa, M. Uncertainty relations for noise and disturbance in generalized quantum measurements. Ann. Phys. 311, 350-416 (2004).

14. Ozawa, M. Universal uncertainty principle in measurement operator formalism. J. Opt. B 7, S672-S681 (2005).

15. Shull, C. G. Single slit diffraction of neutrons. Phys. Rev. 179, 752-754 (1969).

16. Yuen, H. P. Two-photon coherent states of the radiation field. Phys. Rev. A 13, 2226-2243 (1976).

17. Breitenbach, G., Schiller, S. \& Mlynek, J. Measurement of the quantum states of squeezed light. Nature 387, 471-475 (1997).

18. Appleby, D. M. The error principle. Int. J. Theor. Phys. 37, 2557-2572 (1998).

19. Werner, R. F. The uncertainty relation for joint measurement of position and momentum. Quant. Inf. Comput. 4, 546-562 (2004).

20. Busch, P., Heinonen, T. \& Lahti, P. Heisenberg's uncertainty principle. Phys. Rep. 452, 155-176 (2007).

21. Klepp, J. et al. Observation of nonadditive mixed-state phases with polarized neutrons. Phys. Rev. Lett. 101, 150404 (2008).

22. Koshino, K. \& Shimizu, A. Quantum Zeno effect by general measurements. Phys. Rep. 412, 191-275 (2005).

23. Mir, R. et al. A double-slit 'which-way' experiment on the Complementarity-uncertainty debate. New J. Phys. 9, 287 (2007).

24. Lund, A. P. \& Wiseman, H. M. Measuring measurement-disturbance relationships with weak values. New J. Phys. 12, 093011 (2010).

25. Williams, G. Polarized Neutrons (Oxford Univ. Press, 1988).

26. Yuen, H. P. Contractive states and the standard quantum limit for monitoring free-mass positions. Phys. Rev. Lett. 51, 719-722 (1983).

27. Ozawa, M. Measurement breaking the standard quantum limit for free-mass position. Phys. Rev. Lett. 60, 385-388 (1988).

28. Maddox, J. Beating the quantum limits. Nature 331, 559 (1988).

29. Nielsen, M. A. \& Chuang, I. Quantum Computation and Quantum Information (Cambridge Univ. Press, 2000).

30. Kraus, K. States, Effects, and Operations: Fundamental Notions of Quantum Theory (Lecture Notes in Physics, Vol. 190, Springer, 1983).

\section{Acknowledgements}

We acknowledge support by the Austrian Science Fund (FWF), the European Research Council (ERC), the Japan Science and Technology Agency (JST) and The Ministry of Education, Culture, Sports, Science and Technology (MEXT) in Japan. We thank H. Rauch, M. Arndt (Vienna) and A. Hosoya (Tokyo) for their helpful comments.

\section{Author contributions}

J.E., G.S. and S.S. carried out the experiment and analysed the data; G.B. contributed to the development at the early stage of the experiments; M.O. supplied the theoretical part and conceived the experiment; Y.H. conceived and carried out the experiment; all authors co-wrote the paper.

\section{Additional information}

The authors declare no competing financial interests. Reprints and permissions information is available online at www.nature.com/reprints. Correspondence and requests for materials should be addressed to Y.H. 


\section{CORRIGENDUM}

\section{Experimental demonstration of a universally valid error-disturbance uncertainty relation in spin measurements}

Jacqueline Erhart, Stephan Sponar, Georg Sulyok, Gerald Badurek, Masanao Ozawa and Yuji Hasegawa

Nature Physics 8, 185-189 (2012); published online 15 January 2012; corrected after print 12 March 2012.

In the version of this Letter originally published, in Fig. 5a a factor of $\hbar / 2$ appeared incorrectly against the label 'Heisenberg lower limit' and in the figure caption. This error has been corrected in the HTML and PDF versions of the Letter.

\section{CORRIGENDUM}

\section{Experimental demonstration of a universally valid error-disturbance uncertainty relation in spin measurements}

Jacqueline Erhart, Stephan Sponar, Georg Sulyok, Gerald Badurek, Masanao Ozawa and Yuji Hasegawa

Nature Physics 8, 185-189 (2012); published online 15 January 2012; corrected after print 12 March 2012 and 1 August 2012.

In the version of this Letter originally published, in the Methods section under the heading 'Error and disturbance in spin measurements: theoretical determination' the equation defining $\eta(B)$ was incorrect - the term $\sigma_{\phi}$ should have been divided by 2 as shown below. This error has been corrected in the HTML and PDF versions of the Letter.

$$
\eta(B)=\sqrt{2} \|\left[\sigma_{\phi} / 2, \sigma_{y}\right]|\psi\rangle \|=\sqrt{2} \cos \phi
$$

